\title{
The Cellular Pathways of Liver Fibrosis in NASH
}

\author{
Méndez-Sánchez N 1,2*, Coronel-Castillo CE ${ }^{1}$, Contreras-Carmona J ${ }^{1}$, Barranco-Fragoso $\mathrm{B}^{3}$ and \\ Qi X ${ }^{4}$
}

1 Liver Research Unit, Medica Sur Clinic \& Foundation, Mexico City, Mexico

2 Faculty of Medicine, National Autonomous University of Mexico, Mexico City, Mexico

3 Department of Gastroenterology, National Medical Center "20 Noviembre", 03229 Mexico City, Mexico.

4 General Hospital of Northern Theater Command, Department of Gastroenterology. Shenyang, Liaoning 10016 China

* Correspondence: Prof. Nahum Méndez-Sánchez, MD, MSc, PhD. Liver Research Unit, Medica Sur Clinic and Foundation, Puente de Piedra 150, Col. Toriello Guerra, ZP. 14050, México City, México. Email: nmendez@medicasur.org.mx

Abstract: Nonalcoholic steatohepatitis (NASH), which is characterized by liver steatosis, inflammation and fibrosis, is the most severe variation of nonalcoholic fatty liver disease (NAFLD). This disease is a consequence of several metabolic alterations such as type 2 diabetes and dyslipidemia that trigger different pathways of cell dysfunction and systemic inflammation which ultimately affect the liver. Furthermore, those mechanisms activate a complex cascade of immune response after repeated cell aggression. In the liver cytokines and interleukins interact with network of innate immune cells, including Kupffer cells (KCs), dendritic cells (DCs), lymphocytes and hepatic stellate cells (HSC). These cells translate those signals into immune responses and pathologic hepatic changes during the development of NASH. In this scenario the development of fibrosis is the most important change since it is an adaptive mechanism that in the short time has the objective of repair the damaged tissue but after prolonged injury it progresses to parenchymal scarring, cellular dysfunction and finally to organ failure. Finally, since NASH is an important cause of liver cirrhosis; this review addresses the cellular pathways of fibrosis in the setting of NASH explained by the interaction between immune and hepatic cells.

Keywords: liver fibrosis; NASH; innate immune cells.

\section{Introduction}

The liver is one of the most regenerative tissues in the body and is capable to regenerate itself even after partial hepatectomy. But despite its unique characteristics and the extraordinary capacity to regenerate upon various injuries, there is critical difference in the response to transient or chronic damage (1). Usually, after acute injury, the liver will be able to return to its original architecture by proliferation and remodeling of the remaining cells within weeks trough the interaction of cytokines and interleukins with a network of innate immune cells, including KCs, DCs, lymphocytes and HSC. The complex cascade of cell interaction will result in the removal of unwanted cells and the proliferation of new cells by growth factors such as Hepatocyte Growth Factor (HGF) or the Transforming Growth Factor Alpha (TGF alpha) $(2,3,4)$. The balance between the immune response, the grade of apoptosis, that should be equal the grade of cells generated by mitosis, and the grade of liver injury are important factors to recover a healthy hepatic tissue $(4,5)$. In contrast to acute liver injury, chronic injury overcomes the regenerative capacity of the liver resulting in fibrosis instead 
new tissue. Regarding this, fibrosis is an adaptative mechanism that in the short time has the objective of repair the damaged tissue but after prolonged injury it progresses to parenchymal scarring, cellular dysfunction and finally to organ failure (6). In NASH, there is a dysregulation of physiological remodeling, overactivation of hepatic stellate cells, activation of myofibroblasts, and formation of a fibrous scar (Figure 1). Specifically, for NASH, the chronic injury comes from several metabolic alterations that trigger different pathways of cell disfunction and systemic inflammation. Furthermore, the immune response is related to cell damage caused by metabolic stress which is present in several diseases such as Type 2 diabetes (T2D), obesity and even blood hypertension. Moreover, the potential immune response against cell damage in the scenario of metabolic syndrome (MetS) could be altered since the regulators of immune-metabolic interactions include host genetics, nutritional status, and the intestinal microbiome (7).

Besides, due to global impact of NASH and the lack of accurate pharmacological treatment, the study of the cellular and molecular pathways underlying this disease becomes important (8). The later becomes clear just by following the different trials of many drugs with molecular targets ranging from improving metabolic mechanism in hepatocytes to antifibrotic therapy (9). Moreover, the impact of liver fibrosis affects other important systems that are affected by MetS and NASH $(10,11)$. Regarding this, the liver is an important regulator of many metabolic functions and is also the cause of many alterations in other systems, mainly cardiovascular, it has been reported epidemiological associations between NAFLD and organ dysfunction and fibrosis in kidney, heart and systemic vessels; not to mention that liver fibrosis shares many common pathways of fibrosis with other organs such as lungs and pancreas. $(12,13,14)$ Finally, due to many mechanisms of NASH causing liver cirrhosis; this review addresses the cellular pathways of fibrosis explained by the interaction between metabolic alterations, immune cell and the regenerative mechanism of the liver. In NAFLD the origin of liver damage comes from outside the liver such as in adipose tissue (AT) and gut or inside the liver due to lipotoxicity, innate immune response and cell death pathways (15).

\section{Overview of hepatic cells response to damage}

Cells are everyday under constant damage, most of them do not represent a significant stress to cause cell dysfunction. Usually, the commons threats of cytotoxic and mutagenic effects of DNA damaging come from agents that we can find in poor quality food, infections and ionizing radiation. But there is a wide variety of endogenous agents that cause DNA damage, such methylating species and the reactive oxygen species (ROS) (16). Still, the cells are capable to deliver its genetic material, intact and unchanged, to the next generation (17). Even if the cell fails in its attempt to repair the DNA, there is one last mechanism which is part of its own cycle of life and is named apoptosis. The capacity to eliminate senescent, damaged, genetically mutated, or virus infected cells, is crucial for the maintenance of a healthy tissue and this occurs also in the liver. The removal of damaged cells decreases the release of proinflammatory cytokines and cause a minimal immune response. Paradoxically, chronic injury and chronic apoptosis increases the risk of mitotic errors. Even more, high levels of apoptosis have been identified in nearly all types of fibrosis, and many mechanisms by which the apoptotic cells might dictate fibrotic outcomes (Figure 1) $(4,18)$.

Nevertheless, the death of damaged cells by apoptosis and the creation of new ones by mitosis is not that easy. When there is injury in a certain tissue, its cells release a cascade of molecules with two objectives 1) Activate immune cells to "control the damage" and 2) Repair the tissue. Those molecules include histones and interleukins from the cell nucleus, cyclophilin A and uric acid crystals form the cytosol among others and known as damage-associated molecular patterns (DAMPs) (19). In recent years DAMPs play a key role in many mechanisms of different diseases. Before DAMPs were described, the molecules involved in immune host cells activation were known as pathogenassociated molecular patterns (PAMPs) which are present in the bacterium and are also recognized by the immune system (20). Regarding NASH, the alteration of metabolic functions damages the hepatic cells with a consequent release of DAMPs but also PAMPs due to dysbiosis and activation of inflammatory mediators. Each one of the hepatic cells play a key role in the response to those 
metabolic alterations by disruption of the hemostasis of liver reparations and leading to abnormal fibrosis and the first of those cells is no other than the Hepatocyte.

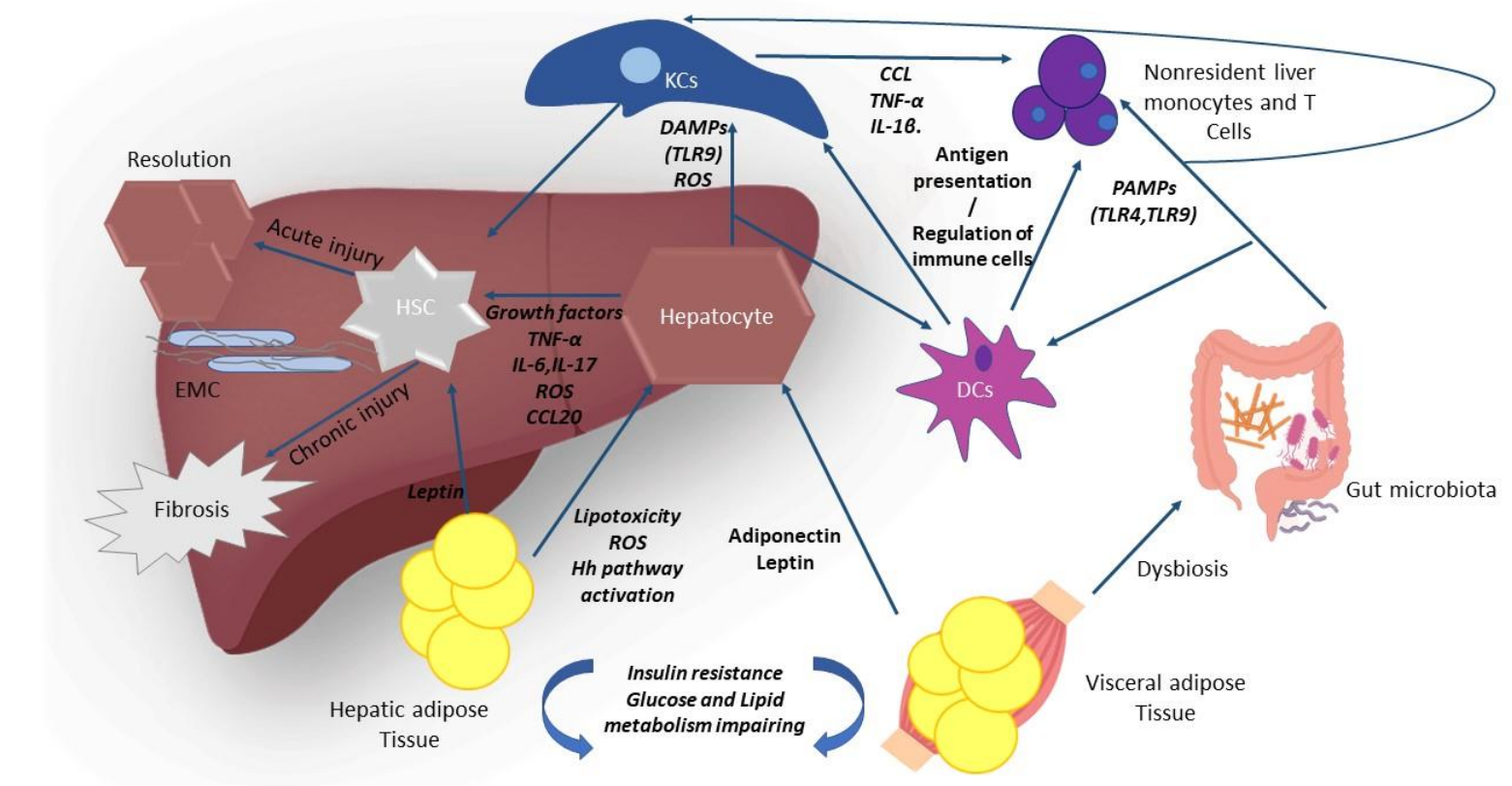

Figure 1. Balance between liver regeneration and fibrosis according with the multiple hit model in NASH. A high-fat diet increases the visceral adipose tissue which is the cause of hepatic steatosis. In this context the failure to metabolize and to storage will cause lipotoxicity; therefore, this first hit of cellular damage mainly in hepatocyte is followed by multiple hits and multiple injuries coming from different sources. Lipotoxicty can affect also Kupffer cells (KCs) and Hepatic stellate cells (HSC), and all three cells can release a myriad of interleukins and chemokines in response to the activation of each one. In parallel, lipids can alter gut microbiota and induce dysbiosis which adds more proinflammatory ligands to KCs and HSC. The production of ROS either by lipids and glucose metabolism or direct cell damage are recognized as DAMPs and again this can contribute to inflammation. If all those insults to the liver stop in the acute phase, HSC are capable to repair the damaged tissue, remove senescent cells and by the deposition of extracellular matrix (ECM) create healthy tissue. In contrast, the overwhelming chronic damage exceeds the HSC capacity to recover functional tissue and instead of that, EMC will transform into fibrosis and scarring bridges among cells, being that a hallmark of NASH. Furthermore, Dendritic cells (DCs) seem to play an interesting role since these cells have ability to capture some molecules from PAMPs and DAMPs to present as antigen to KCs and other immune cells such as T cells, according to that for some mechanism that until now it not well understood DCs can prevent to liver fibrosis by attenuating the grade of inflammatory response.

\subsection{Hepatocyte}

NASH is characterized by the accumulation of fat in the liver in parallel with hepatocyte damage, inflammation, and different degrees of scarring or fibrosis, and has a high risk of progressing to cirrhosis and hepatocellular carcinoma (21). As stated before, liver fibrosis is the result of the woundhealing response of the liver after repeated injury, especially against hepatocyte where they regenerate and replace the necrotic or apoptotic cells. This process is associated with an inflammatory response and a limited deposition of extracellular matrix (ECM). As fibrotic liver diseases advance, disease progression from collagen bands to bridging fibrosis to frank cirrhosis occurs $(4,22)$.

The hepatocytes are the liver parenchyma, they represent around 85\% of the liver mass (23). These cells can be damaged by direct and mechanical injury. For example, in cholestatic diseases toxic bile acids (BAs), can cause insults over the hepatocyte's membranes, induce apoptosis and activate the NF- $\kappa$ B pathway (24). Still, in most instances, liver injury occurs because of immune-mediated or direct injury to the hepatocytes. 
In the context of NAFLD and NASH, the aggression against hepatocytes can be explained trough the classic "two-hit" theory where the first hit is represented by the accumulation of Free Fatty Acids (FFAs), Triglycerides (TGs) and free cholesterol (FC) which induced a second hit with the activation of inflammatory response (25) Regarding this, the injury in hepatocytes initiated since the first hit because FFAs, particularly saturated ones such as palmitate seems to be lipotoxic and can induce endoplasmic reticulum (ER) stress through increased saturation of membrane phospholipids, alter mitochondrial metabolism and promote ROS production, and induce insulin resistance as well as inflammation (Figure 1) (26). For the case of TGs, their accumulation may not be the initial cause of NAFLD development, but they play an important role in the early adaptive response to increased lipid overload, particularly to saturated FFAs. This can be also observed during the natural history of NAFLD since liver biopsy will show steatosis, lobular inflammation, and ballooned hepatocytes and fibrosis which all are necessary components for the diagnosis of NASH (27).

Furthermore, an increase of beta oxidation of FFAs and the production of ROS, like Hydrogen peroxide induces damage over mitochondrial DNA (mtDNA). Recently, another mechanism of DNA damage is the sirtuin imbalance due hepatic glucose and fatty acid metabolism (28). In fact, patients with NAFLD have a decreased expression of different sirtuin proteins such as SIRT1, SIRT3, SIRT5, and SIRT6. This is associated with increased expression of lipogenic genes including sterol regulatory element binding protein-1 (CREB1), fatty acid synthase, and acetyl-CoA carboxylase (29). On the other hand, ROS act as a second messengers and activates mitogen-activated protein kinase (MAPK) and nuclear factor $\kappa \mathrm{B}$. In addition, not only Adenosine triphosphate (ATP), Uridine triphosphate (UTP), uric acid, High mobility group box 1 (HMGB1) or ROS that come from an injured hepatocyte are part of DAMPs, even its own mtDNA can be a DAMP as ligand to toll-like receptor (TLR) which are widely present in innate immune cells such as monocytes and KCs (30).

Those signals can even induce necrosis and apoptosis conforming other DAMPs that activate also HSC within the liver. These activated cell populations are key mediators of the regenerative or fibrogenic response (Figure2) (31). Furthermore, TLR activate the transcriptions factors JNK and NF$\kappa \beta$ leading to the expression of cytokines such as tumor necrosis factor- $\alpha$ (TNF $\alpha$ ), IL-1 $\beta$ and IL-6, which contribute to the progression of NAFLD; this correlates with several studies where mtDNA levels are elevated during liver injury or there is a loss of mtDNA mechanisms of adaptation (31). In a study in mice with NASH, investigators found that mtDNA from hepatocyte are specific ligands to TLR9 (32).

Finally, while the two hit model can easily explain the chain of activation and response between hepatic cells, the "multiple hit model" is accepted theory in NASH because it involves a widespread metabolic dysfunction and an interaction with genetic and environmental factors as well as changes in crosstalk between different organs beyond the liver, including AT and de dysbiosis in the gut (25). 
Figure 2. A cross talk between liver cells and their response to different stimuli. Major cells involved NASH are Hepatocytes, the main victim of many insults, KCs and other immune cells like DCs play a key role in the response to such insults and HSC activation are de conclusion of all these events since these cells are the effector cells in either regeneration or liver fibrosis. Yet, to response to so many stimuli, all those cells express a myriad of receptors and there is an Intime crosstalk between them. In NASH FFAs and excess of TGs induces lipotoxicity in the three major hepatic cells but mainly hepatocytes. Inside this cell, toxic lipid traffic, beta oxidation intermediaries and ROS from other altered metabolic pathways induced ER stress and Mitochondrial damage. At the same time, the type of diet in NASH patients induced gut dysbiosis which will cause an alteration in BAs composition due to bacterial translocation that additionally will release PAMPs in form of LPS to activate KCs and other immune cells. Furthermore, FCs detect both PAMPs from dysbiosis increasing liver inflammation and damaging hepatocytes and activating HSC trough CCL20, interleukins and TNF$\alpha$. Paradoxically, the combination of lipotoxic and the activation of KCs by gut dysbiosis and even lipotoxicity itself active de MAPK and NF- $\kappa$ B pathways, and alters de UPR inducing apoptosis in the hepatocyte. Eventually, the senescent hepatocyte can release growing factors to active HSC but also Hh ligands and ROS that again will induce ER stress and mtDNA damage but now in the HSC. Interestingly, this kind of feedback response, where these three cells can be activated by the other two during liver damage and response to promote a more proinflammatory state, created a vicious circle. If that was not enough, the altered BAs serve as antagonist to FXR an impairs de functions of PPAR and then alters lipid and glucose metabolism, perpetuating the process of lipotoxicity and metabolic abnormalities that the patients with NASH already have. Finally, DCs are capable to attenuate the inflammatory response that also can damage PPAR receptors and its functions. Nevertheless, DCs instead of attenuate inflammatory response can also increase it promoting indirectly fibrosis but also directly by activating HSC.

\subsection{Kupffer Cell}

Innate immune mechanisms are the most important drivers of inflammation and other pathological manifestations observed in NASH including steatosis, insulin resistance (IR), and fibrosis. These cells can sense excess metabolites and translate those signals into immune responses and pathological hepatic changes during the development of NASH (33).

KCs are the resident liver macrophages and they are a critical component of the mononuclear phagocytic system and are central to both the hepatic and systemic response to pathogens (34). Dysregulation in the control of inflammatory responses in KCs can contribute to chronic inflammation in the liver (35). Furthermore, the role of KCs is interesting since it has alternative roles 
as protector or as damage mediator. In a study by Huang et al. the depletion KCs prevented the development of diet-induced hepatic steatosis and insulin resistance (36). This can be explained since lipid metabolism is a general function of macrophages which is executed by transcription factors such as Liver $\mathrm{X}$ receptor $\alpha(\mathrm{LXR} \alpha)$, Retinoid $\mathrm{X}$ receptor $\alpha(\mathrm{RXR} \alpha)$ and Peroxisome proliferator-activated receptors $\delta$ and $\gamma$ (PPAR $\delta$, PPAR $\gamma$ ) that are also present in KCs (37). It is important to notice that this mechanism, among others, is not the cause of NASH but consequence. Paradoxically the alterations of lipid metabolism in KCs due to NASH is a mechanism that perpetuate the disease (Figure 2).

But When KCs become a threat to the liver? These cells are activated by molecular signals such as structural motifs of proteins, lipids, and nucleic acids that originate from invading microorganisms [PAMPs] in the case of dysbiosis but also KCs can detect components released from host cells that are injured, dying, or undergoing malignant transformation [DAMPs], those molecules include heat shock proteins, high mobility group box 1 protein, breakdown products of the ECM like hyaluronan, fibrinogen, fibronectin and uric acid but also ligands to TLR such a mtDNA from hepatocytes (30) (38) . In NASH, DNA damage is induced by ROS and lipid metabolism releasing those DAMPs from damaged hepatocytes, this process named lipotoxicity is characterized by an increased concentration of toxic lipids and lipids derivatives. Many types of lipid have been demonstrated to mediate liver lipotoxicity, including FFAs, TGs, FC, lysophosphatidyl-cholines (LPCs) and ceramides (39). At the same time and even without liver damage, the high amounts of FFAs and FC increase the activation state of KCs (40). In addition, a high-fat diet with cholesterol induced hepatic inflammation, foamy KCs in LDL receptor-deficient and the accumulation of cholesterol crystal in KCs. Additionally, phagocytic dysfunction of KC can accelerate inflammatory necrosis during hepatocyte fat accumulation (41).

The activated KCs release proinflammatory cytokines and chemotactic factors such as chemokine C-C motif ligand (CCL), TNF- $\alpha$ and IL-1 $\beta$. In fact, these cytokines can recruit nonresident cells to the liver such as neutrophils, natural killer (NK) T lymphocytes, CD4+ and CD8+ T cells (38). In fact, recent studies have brought to the table the discussion around that different macrophage populations are present in the liver in health and disease. In 2016, Reid et al found in an experimental model of steatohepatitis in mice, changes in the recruitment of multiple immune cells including CD4+ and CD8+ T cells, NK cells (42). Also, they described that Clodronate treatment resulted in the reduction of infiltrating CD8+ T cells likely contributing to further protection against hepatocellular injury as increased portal CD8+ T cells has been observed in patients with NASH. The later was previously described in at least two studies where the suppression of KCs with Clodronate or gadolinium improve the liver damage $(36,43)$.

On the other hand, KCs promote other pathways of liver injury beyond its chemotactic and cytotoxic functions. The expression of complement components such as $\mathrm{C} 1$, the chemokine CCL and the interleukins IL-15 and IL-1beta suppressed the PPAR $\alpha$ expression (44). This is especially important since PPAR $\alpha$ plays a critical role in the regulation of fatty acid uptake, beta oxidation, ketogenesis, bile acid synthesis, and triglyceride turnover.

Regardless of the mechanism by which KCs induce liver damage in NASH and its unregulated function that perpetuates metabolic alterations, the final consequence of KCs signaling functions is fibrosis by activation of HSC.

\subsection{Hepatic Stellate Cell}

After a long and complex cascade of molecular signaling by both KCs and Hepatocytes, fibrosis will lie in the HSC functions.

In normal conditions, myofibroblasts are absent from liver tissue. At acute injury state, these cells restore the liver parenchyma by forming a mechanical scar that is usually dissolved when the tissue is repaired. At this stage myofibroblasts are cleared by apoptosis or by inactivation. In contrast, in chronic liver injury, myofibroblast activation causes the accumulation ECM consequently leading to fibrosis (45). Regarding this, myofibroblast are no other than liver resident mesenchymal cells 
which in fact are HSC and portal fibroblasts (PFs). Although the composition of fibrogenic myofibroblasts varies dependent on etiology of liver injury $(45,46)$.

In any case, models in mice exposed to hepatotoxic damage showed that HSC are the prominent cells in chronic injury, as it happens in NASH (47). Under physiological conditions, HSC exhibit a quiescent phenotype and serve as a major storage of Vitamin A. These quiescent HSCs (qHSCs) response to stimuli from platelet-derived growth factor $\beta$ (PDGF $\beta$ ), transforming growth factors, especially (TGF $\beta$ ) and connective Tissue Growth Factor (CTGF). Thus, qHSCs rapidly downregulate Vitamin A-containing lipid droplets and neural markers and differentiate into collagen Type I and $\alpha$ smooth muscle actin $(\alpha \mathrm{SMA})$, In contrast they upregulate production of matrix metalloproteinases MMPs $(46,48)$. Alternatively, the auto-activation of HSC by $\beta$-PDGFR is related with accelerated fibrosis (49).

Besides, HSC expresses a myriad of receptors for different molecules that upon stimulation promote differentiation of this cell type to cellular matrix-production cell (Table 1).

For instance, patients with NASH have a high level of leptin in contrast with adiponectin. The first adipokine has high profibrotic effect (50). Leptin can be secreted via KCs and other nonparenchymal cells in the liver to HSC signal transduction where it affects the perpetuation phase of the activated HSC life cycle. Even more, leptin first is a potent mitogen for HSC. In both in vivo and in vitro studies leptin promotes HSCs into the M phase of the cell cycle; and, is nearly as potent a mitogen as PDGF (51). On the other hand, the role of interleukins secreted by KCs are also important for HSC differentiation; in this case TNF- $\alpha$ and Il-6 especially important. About this, Liu et al found in rats with liver fibrosis that the areas with more KCs activity as well as more expression of TNF- $\alpha$ had also an important number of $\alpha$-SMA- and collagen type I-positive cells that interestingly do not underwent apoptosis (52). These findings correlate with transcriptional activity that oxidative-stress and IL-6 mediate to induce fibrosis (53). Alternatively, the C-C motif chemokine ligand 20 (CCL20) which is highly up-regulated transcript in NAFLD-associated fibrosis seems to be released by HSC. This means that HSC are also capable to induce fibrosis by themselves. Furthermore, CCL20 is produced by stellate cells in response to lipid loading may therefore be key mechanism in the fibrotic progression of NAFLD in response to the increased caloric intake in extreme obesity (54). Another chemokine that is expressed in NASH, particularly in early stages, is Ccl5. In a study where mice were fed with a choline-deficient, L-amino acid-defined and high fat diet for three weeks they develop steatohepatitis and showed an increase in Ccl5 secreted by HSC, at this point the chemokine induced steatosis and pro-inflammatory factors in hepatocytes through the receptor Ccr5 (55).

Seeing that HSC cab be activated by several ways, is important to highlight how those signals are translated inside the cells. In order to integrate all stimuli that HSC receives, two main transcription factors, the SMAD3 and STAT3 are staged (56). It is known that for instance TGF $\beta$ phosphorylates SMAD3 (Figure 2) (57). Yet, recently some studies have proposed new transcriptional regulations in the context of NASH.

Table 1. Cells and molecules involved in HSC activation

\begin{tabular}{cc}
\hline Cell & Molecule \\
\hline & CTGF \\
Hepatocytes & PDGF $\beta$ \\
KCs & TGF $\beta$ \\
T Cells & VEGF \\
DCs & Interleukins and TNF- $\alpha$ \\
Neutrophils & Leptin and adiponectin \\
Adipocytes & PAMPs (TLR) \\
& DAMPs \\
& ROS
\end{tabular}


factor; PAMPs: pathogen-associated molecular patterns, TLR: toll-like receptor, DAMPs: damage-associated molecular patterns, ROS: reactive oxygen species

Therefore, Marcher et al conducted a study to determinate the changes in HSC transcriptome during development of western diet and fructose-induced NASH in mice; as a result of that, western diet also induced hepatic $\alpha$ SMA expression and fibrosis and HSC mRNA was sequenced. Finally, they identify that ETS1 and RUNX1 TF were significant predictors of HSC gene induction in NASH and early fibrosis (48). Both heterodimers alter the DNA conformation. Besides, others ligand can induce transcriptional regulation in HSC, this is the case of Guanine nucleotide-binding $\alpha$-subunit 12 (G $\alpha 12$ ) which is upregulated in HSC as a consequence of the dysregulation of a specific microRNA that is abundant in these cells, facilitating the progression of liver fibrosis by promoting autophagy (58).

Finally, we can summarize that HSC important cells in the development of NASH. They are the major cells in fibrosis and are capable to respond to KCs and hepatocytes stimulation. Even more, HSC can release several transcriptional factors for itself and other cells in response to chronic liver injury. The role of HSC in NASH has become such important that a myriad of studies about myofibroblasts are gaining importance in different chronic liver diseases (45). Even more, clinical trials in NASH include direct antifibrotic therapy, being Cenicriviroc the first antifibrotic drug for this disease. The CCR5 inhibitory component of Cenicriviroc blockades the inflammation and fibrogenic activation of HSC (59).

\subsection{Dendritic cells}

In recent years, a nonparenchymal liver cell has come to light as an important mediator in liver fibrosis, including NASH-induced fibrosis due to their role as crosstalk between KCs, nonresident liver immune cells and HSC, these cells are Dendritic cells (DCs). Though there is contradictory data among different studies about its true importance in liver fibrosis (60). For example, there is no doubt that DCs have a migratory capacity and a remarkable ability to produce proinflammatory cytokines and they paly a key role in antigen presentation to $\mathrm{T}$ cells, but also those cytokines can activate resident liver cells and induce fibrosis in the context of disease (61). Regarding this, Connolly et al reported that the depletion of DCs inhibited the expression of inflammatory mediators, such as IFN$\gamma$, IL-1 $\beta$, IL-13, in non-parenchymal cells from fibrotic livers of CD11c-DTR mice treated with TAA/leptin (62).

In contrast, Pradere and cols observed in mice that macrophages but not DCs promoted survival of activated HSC via activation of NF- $\mathrm{BB}$ by TNF $\alpha$ and IL-1. That was related with the increasing in fibrosis (63).

The above can be explained by NASH models that are used in mice since in both mice and Humans, there are two distinct populations of hepatic DCs according with their lipid content. Those Lipid-rich hepatic DCs are highly immunogenic and produce TNF while lipid-poor DCs are rather tolerogenic and able to induce CD4 T cells activation (64). In addition, mature DCs, which are expressed in chronic liver damage, facilitate the deposition of monocytes and activation of HSC (61).

To complicate this debate, DCs are also involved in the regression of fibrosis after liver injury since these cells can induce differentiation of Th17 T cells, the main producers of IL-7 which are associated with hepatic steatosis and proinflammatory responses in NAFLD, accelerating the transition from simple steatosis to NASH. Furthermore, studies in liver injured rodent model using carbon 
tetrachloride revealed fibrosis regression after cessation of insult in an environment depleted of DCs. Moreover, those models also showed that fibrosis regression its not only trough HSC since it was found that DCs limit CD8 ${ }^{+}$T-cell expansion and restrict TLR expression and cytokine generation from KCs, neutrophils, and monocytes in NASH (61). Basically, DCs play a key role in the balance of immune cells in hepatic environment $(60,65)$.

\subsection{T Cells}

Innate cells are not the only ones that play a role in NAFLD and NASH. In addition to the inflammatory environment in the liver, the adaptive immune system is involved in obesity-related AT inflammation and the pathogenesis of NAFLD and each one of T Cell type interacts in different ways. Still, the antigen recognition either PAMPs or DAMPs is achieved through the specialized Tcell receptor (TCR), which is composed of an $\alpha$ and $\beta$ protein in most of T cells and the CD3 complex (65). Moreover, animal models with fructose induced NAFLD and deficient T Cells do not develop the disease while immunocompetent mice with effective T Cells and NK cells do (66).

On top of that, CD4 (Helper T Cells) and CD8 (Cytotoxic T Cells) are the main T cells in NASH. Functionally, CD4 T cell responses in NASH are skewed toward Th1 and Th17 phenotypes. Th1 responses in NASH are characterized by secretion of IFN- $\gamma$ and TNF- $\alpha$, which in turn help polarize macrophages toward M1 responses (67). In addition, Th1 is highly present in visceral AT as it happens in most patients with NASH (65) and are related with susceptibility to oxidative stressinduced apoptosis facilitating the transformation of simple steatosis into steatohepatitis (68).

For the case of Th17, the production of IL-17 plays a critical role in the pathogenesis of liver fibrosis through hepatic stellate cell activation (69) but also DCs (65). Moreover, IL-17 axis playing a broad role in multiple models of NAFLD via modulation of hepatic inflammation whereas HSC, KCs, hepatocytes and endothelial cells express receptors for IL-17RA and are known to activate inflammatory pathways which exacerbate the disease (70).

On the other, despite that several studies proved that CD8 are present in NAFLD and their inhibition in animal models decrease in liver steatosis, liver inflammation and transaminases, their mechanism remain unclear $(60,65)$.

\section{NASH}

As it was stated before, NASH can be explained by multiple parallel-hit model as a result of an interaction between environmental and genetics factors as well as a myriad of intrahepatic and extrahepatic insults. $(25,28)$. However, the primary defect in NASH is mitochondrial dysfunction and the main source of mtDNA is oxidative stress $(28,71)$ which is caused by an excessive oxidation of many macromolecules, in this case lipids as response of their accumulation. Even more, excess of visceral AT increases a proinflammatory state (72).

Notwithstanding, oxidative stress and immune activation by an excess of AT in the liver, NASH alters the homeostasis outside the liver. In fact, patients with NASH typically have dysbiosis which means an imbalance in composition of microbiota that has a negative effect on the physiology of the host (73). Dysbiosis in NASH patient impacts the metabolism of substances acquired in diet and increases the presence of PAMPs from bacterium thereby it impacts the cellular response in the liver (74).

On the other hand, MetS in NASH tend to be worse revealing it as part of a continuum of metabolic pathogenesis. In fact, in patients with NASH and obesity, the accumulation of lipid molecules, the type of those molecules and how they are metabolized are the main cause of metabolic disfunction. Dietary lipids, lipolysis of AT and de novo lipogenesis contribute to the pool of lipids stored in the liver. Furthermore, saturated fat intake is correlated with an impaired glutathione metabolism towards oxidative stress leading to NAFLD progression. In fact, saturated lipids fats diet is related with the upregulation of PNPLA3 which is representative gene in NAFLD which is related with lipid metabolism. On the other hand, carbohydrates are important in the progression of the 
disease. For instance, the ingestion fructose from soft drinks in patients with NASH is associated with more advanced liver fibrosis $(75,76)$. Nevertheless, insulin resistance is typically the main metabolic dysfunction in patients with NASH. Insulin resistance is often associated with chronic low-grade inflammation, and numerous mediators released from immune cells and adipocytes may contribute liver damage and liver disease progression (Figure 2) (76).

In addition, the release of FFAs from dysfunctional and insulin-resistant adipocytes results in lipotoxicity, which is caused by the ectopic accumulation of triglyceride-derived toxic metabolites and the subsequent activation of inflammatory pathways, cellular dysfunction, and lipoapoptosis (39).

\subsection{Lipotoxicty and insulin resistance}

It is well known that lipotoxicity is related to insulin resistance and hepatic steatosis. Nowadays, the development and progression of NASH is mainly viewed as the consequence of liver lipotoxicity that occurs when the liver capacity to utilize, store, and export FFAs and TGs is overcome by FFAs flux from the periphery or hepatic de novo lipogenesis (39).

To understand the process of lipotoxicity and how it induces fibrosis, is necessary to recognize the mediators. First, steatosis is an adaptative response to handle excess FFAs, hence the accumulation of TGs droplets was recognized as a first step in NAFLD. Yet, recent studies report that the formation of lipid droplets may be a protective response that prevents lipotoxicity from other fatty acid-derived species. Regardless, TGs storage as fat droplets is only a temporizing measure and if the cell is still unable to handle them appropriately through other metabolic pathways, the stored triglyceride could still serve as a source of lipotoxic intermediates (77).

Regarding the latter, several experimental data also suggests that saturated FFAs are hepatotoxic, while unsaturated FFAs are not injurious to the liver. In fact, in experimental models of obesity and steatosis in animals, where leptin receptor-deficient mice, were fed methionine and choline-deficient diet, hepatic steatosis, apoptosis, ROS production, and consequently fibrosis increased inversely to TG accumulation (78).

In addition, bot FFAs accumulation and insulin resistance (see below) will cause ER stress, a hallmark of NASH psychopathy. Nevertheless, the liver have the ability to response to chronic ER stress through an adaptive signaling pathway known as the unfolded protein response (UPR) (79). This mechanism is critical to maintain the main ER function, protein homeostasis, and it is activated only under stress conditions, since in such terms the normal ER function becomes compromised leading to the accumulation of unfolded proteins. If the UPR fails, there is no other solution that induce apoptosis in the hepatocyte (80).

Even so, before reaching apoptosis, the UPR can cause inflammation and inflammasome activation because the three ER transmembrane receptors involved in this mechanism, PKR-like ER kinase (PERK), activating transcription factor 6 (ATF6 $\alpha$ ), and inositol requiring enzyme 1 alpha (IRE1 $\alpha$ ) are impaired due to hepatic lipid metabolism $(79,80)$. For instance, the PERK pathway activates the transcription factors $\mathrm{C} / \mathrm{EBP} \alpha$ and $\mathrm{C} / \mathrm{EBP} \beta$, which regulate hepatic lipogenesis while the activation of ATF6 $\alpha$ has also been shown to associate with sterol regulatory element binding protein 2 (SREBP2) thus inhibiting its transcriptional activity, which may reduce hepatic lipid accumulation (80).

On the other hand, it is important not to lose sight of the potential mechanism of lipotoxicity to indirectly activate other cells or induce the formation of cytokines by means of mitochondrial damage. As increased formation of ROS increase the formation of lipid peroxidation products and cytokines. In this context TNF- $\alpha$ induces apoptosis in hepatocytes, then TGF- $\beta$, 4-hydroxynonenal (4-HNE) and leptin activate HSC into collagen producing myofibroblasts (81). In contrast, TGF- $\beta$ signaling in hepatocytes also may contributes to apoptosis and lipid accumulation by activation of SMAD proteins and paradoxically induce ROS production that promote the development of NASH (82). Furthermore, the inhibition on TGF- $\beta /$ Smad signaling in diabetic rats reduce liver fibrosis (80)

Finally, another pathway known as the hedgehog $(\mathrm{Hh})$ pathway has been studied widely in recent years since it is involved in the pathogenesis of hepatic steatosis as well as in the progression 
from hepatic steatosis to severe forms of liver damage. The Hh pathway modulates many aspects of cell differentiation and tissue development, its activation or inhibition trough the ligand hedgehog, Patched its receptor, smoothened the signal transducer and the effector transcription factor, Gli, will unleash as cascade of event in hepatocytes and HSC (83). Regarding this, usually in healthy individuals there so few Hh ligands that are surpassed by antagonist ligands. In contrast, patients with NASH express high amounts of Hh ligands in ballooned hepatocytes $(84,85)$. Moreover, an interestingly study by Zhou et al found in 135 human liver biopsies from patients with NASH, that the upregulation of Hh proteins were related with the expression of TGF- $\beta 1$ in activated HSC. Even more, the Hh proteins were related with severity of hepatocellular ballooning, lobular, and portal inflammation (86).

Summarizing the above, lipotoxicity as one of the main mechanisms in NASH inducing fibrosis is the result of the accumulation of lipids that need to be stored and metabolized somehow and, in the process, the resulting of metabolic of intermediates cause cellular damage. In addition, some of lipid molecules are toxic per se. To complicate matter further, the direct or indirect injury over cells release a myriad of ligands and cytokines that will overactivate the immune system.

Alteration in lipids metabolism is a hallmark of NASH while the hormones and cytokines that derive from adipose tissue can lead to insulin resistance as well. Furthermore, as stated before, patients with NASH have high of leptin in contrast to low levels of adiponectin. The latter, not only regulates fatty acid oxidation and inhibits lipid accumulation but also it also maintains whole-body glucose homeostasis, including hepatic insulin sensitivity $(87,88)$. In human the association of hepatic fat and insulin resistance are a direct cause of liver damage and fibrosis but interestingly this can occur without inflammatory pathways that have been widely described in lipotoxicity. Furthermore, insulin resistant hepatocytes exposed to fatty acids can release microparticles that activate HSC promoting ECM deposition (89). Nevertheless, inflammation directly affect glucose homeostasis creating a vicious circle of lipotoxicity, insulin resistance, inflammation and immune alterations and finally increasing more metabolic alterations both lipids and glucose $(90,91)$. If the above was not enough, paradoxically hepatic insulin resistance fails to suppress hepatic glucose production, yet it continues to stimulate lipogenesis (92).

\subsection{Thr role of dysbiosis and dysregulation of immune system.}

The gut-liver axis is still not well understood but there no doubt about the interaction between the Gut and the liver. Dysbiosis could increase permeability of the gut barrier, resulting in translocated bacteria and the products coming from the gut such as gram-negative-derived lipopolysaccharides (LPS) can reach the liver through the portal vein and might lead to increased oxidative stress and inflammation (93).

The type of diet related with NAFLD and obviously obesity can determinate the characteristic of gut microbiota and it could be an independent contributor to the development of NAFLD which can be demonstrated by mouse models of NASH where the increased of some bacterial species, such as Bacteroides spp., showed a positive correlation with altered LPS (94). It is important to notice that LPS are one of the first PAMPs that were recognized, and despite that they were not created in the liver it can be recognized by KCs and unleash a severe immune response in the liver (39).

Regarding the above, a high fat diet can alter the pool of bile acids (Bas) composition, due to the impairs in their metabolism since they are synthetized from cholesterol. Furthermore, livers with that kind of diet have for instance higher glycine-conjugated BAs rather than taurine-conjugated BA which, the alteration in BAs composition is not just a direct consequence of the diet but also is as consequence of gut microbiota dysbiosis because bacteria in the distal small bowel and colon break BAs down into secondary BAs $(95,96,97)$.

Even more, the importance of gut microbiota in BAs metabolism lies in the regulation of farnesoid $X$ receptor (FXR) a nuclear hormone receptor which plays a key role in glucose and lipid homeostasis and even fibrosis. Furthermore, FXR are expressed in HSC being able to directly regulate 
492

493

494

495

496

497

498

499

500

501

502

503

504

505

506

507

508

progression to fibrosis. In addition, FXR can induct the activation of PPAR- $\gamma$ in these cells and inhibit their profibrotic mechanisms (Figure 2)(98).

Several studies about dysbiosis and the alteration showed that bacteria that convert primary bile acids to secondary bile acids are decreased in the faeces of patients with NASH, for example there is a decreasing of Clostridium lentus while there is high population of Enterobacteriaceae which means a reduction in secondary BAs and consequently a reduction in FXR activation. In fact, the change in the pool of BAs increased those that are FXR antagonist. Therefore, new therapies are focus to activate FXR in the liver because its hepatoprotective activities, improving steatosis, inflammation and fibrosis in patients with NASH $(99,100)$.

Regarding to dysregulation of immune system, dysbiosis leads to endotoxemia and inflammation in the liver. This process occurs when the changes in the composition in gut microbiota increases the permeability in the intestinal epithelium by widening of the tight junctions between enterocytes and translocating endotoxins into the portal circulation and subsequently the liver (101). Some of the main PAMPs that comes from bacterial endotoxins are two proteins members of the TLR family known as TLR4 and TLR9, such proteins activate KCs (102) while in HSC, LPS from Gramnegative bacteria promoted hepatic stellate cells mediated fibronectin production through TLR4, and that fibronectin further promoted liver endothelial cells migration and angiogenesis (103).

\section{Conclusions}

NAFLD and its most severe form NASH seems to be diseases of excesses. There are excess of lipids either with or without obesity and there is an accumulation of many metabolic intermediates in both lipids and carbohydrates metabolism. Furthermore, there is an excess of immune response not only in the liver but also in the gut. Hence, fibrosis in NASH is the result of the summarize of many liver injuries that come, at the beginning, from altered metabolic functions which result in the dysregulation of the immune system affecting liver cells as well as nonresident liver cells (Figure 1). Nonetheless, the despite the many insults that the liver receives it is capable to recover its injury tissue and all its functions, the real treat comes when the injury become chronic. In summary, the imbalance between liver regeneration and liver fibrosis is the result of the perpetuation of cell damage and mutual activation. NAFLD will cause lipotoxicity and dysbiosis; therefore, inflammation increases insulin resistance alongside lipotoxicity and those process are pathways to create cellular stress and more inflammation (Figure 2). In this context, the recognition of the different cells involved in NASH is vital in order to implement therapeutic innovations. For instance, until this day, different trials are proving drugs that target cells receptors involving fibrosis, cells receptors that improve metabolic functions and even dysbiosis to attenuate inflammation considering the growing treat that NAFLD and NASH represent.

Author Contributions: N.M.-S.; Conceptualization and methodology, N.M.-S., C.E.C.-C. and J. C.-C investigation and resources, N.M.-S.; data curation, N.M.-S., C.E.C.-C. and J. C.-C writing-original draft preparation, N.M.-S.; writing-review and editing, J. C.-C; visualization, N.M.-S.; supervision SND project administration.

Funding: This research received no external funding

Acknowledgments: We acknowledge the support and invitation to contribute with knowledge to the MDPICells editorial team.

\section{Abbreviations}

NASH: Nonalcoholic steatohepatitis

NAFLD: Nonalcoholic fatty liver disease

KCs: Kupffer cells

DCs: Dentritic cells

HSC: Hepatic stellate cells

HGF: Hepatocyte growth factor

TGF: alpha: Transforming growth factor alpha 
T2D: Type 2 diabetes

543 MetS: Metabolic syndrome

544 AT: Adipose tissue

545 ROS: Reactive oxygen species

546 DAMPs: Damage-associated molecular patterns

547 PAMPs: Pathogen-associated molecular patterns

548 ECM: Extracellular matrix

549 Bas: Bile acids

550 FFAs: Free fatty acids

551 ER: Endoplasmic reticulum

552 CREB1: Element binding protein 1

553 MAPK: Mitogen-activated protein kinase

554 IR: Insulin resistance

555 LXR: Liver $x$ receptor

556 RXR $\alpha$ : Retinoid X receptor $\alpha$

557 PPARס: Peroxisome proliferator activated receptors $\delta$

558 PPAR $\gamma$ : Peroxisome proliferator activated receptor $\gamma$

559 LPCs: Lysophosphatidyl-cholines

560 FFAs: Free fatty acids

561 TGs: Triglycerides

562 FC: Free cholesterol

563 CCL: Chemokine C-C motif ligand

564 TNF- $\alpha$ : Tumor necrosis factor- $\alpha$

565 NK: Natural Killer

566 PDGF $\beta$ : Platelet-derived growth factor $\beta$

567 TGF $\beta$ : Transforming growth factors

568 CTGF: Connective Growth Factor

$569 \alpha$ SMA: $\alpha$ smooth muscle actin

570 CCL20: C-C motif chemokine ligand 20 


\section{References}

1. Tanaka M, Miyajima A. Liver regeneration and fibrosis after inflammation. Inflamm Regen. 2016; $36: 19$

2. Nakamura T, Mizuno S. The discovery of Hepatocyte Growth Factor (HGF) and its significance for cell biology, life sciences and clinical medicine. Proc Jpn Acad Ser B Phy Biol Sci. 2010; 86:588-610

3. Fausto N. Liver Regeneration. J Hepatol. 2000; 32:19-31

4. Guicciardi ME, Gores GJ. Apoptosis: a mechanism of acute and chronic liver injury. Gut. 2005;54:1024-1033

5. Böhm F, Köhler UA, Speicher T, et al. Regulation of liver regeneration by growth factors and cytokines. EMBO Mol Med. 2010; 2:294-305

6. Wick G, Backovic A, Rabensteiner E, et al. The immunology of fibrosis: innate and adaptive responses. Trends Immunol. 2010; 31:110-9

7. Zamora N, Bashiardes S, Levy M, et al. The Role of the Immune System in Metabolic Health and Disease. Cell metabolism 2017;25: 506-521

8. Mahady SE, George J.Predicting the future burden of NAFLD and NASH. J Hepatol. 2018; 69:774-775

9. Oseini A, Sanyal AJ. Therapies in Non-Alcoholic Steatohepatitis (Nash) Liver Int. 2017; 37: 97-103

10. Lee SB. Mechanistic connection between inflammation and fibrosis. Kidney Int Suppl. 2010;119: S22-S26

11. Seki E, Schwabe RF. Hepatic inflammation and fibrosis: functional links and key pathways. Hepatology 2015; 61:1066-1079

12. Patil R, Sood GK. Non-alcoholic fatty liver disease and cardiovascular risk. World J Gastrointest Pathophysiol 2017; 8: 51-58

13. Macuccilli M, Chonchol M. NAFLD and Chronic Kidney Disease. Int. J. Mol. Sci. 2016;17: 562

14. Francque SM, Van der Graaff D, Kwanten WJ. Non-alcoholic fatty liver disease and cardiovascular risk: Pathophysiological mechanisms and implications. J Hepatol 2016; 65:425-443

15. Schuster S, Cabrera D, Arrese M, et al. Triggering and resolution of inflammation in NASH. Nat Rev Gastroenterol Hepatol. 2018; 15:349-364

16. Norbury CJ, Hickson ID. Cellular responses to DNA damage. Annu Rev Pharmacol Toxicol. 2001; 41:367401

17. Sáez GT. Liver Damage and Repair. Int J Mol Sci. 2018;19: 1902

18. Johnson A, DiPietro LA. Apoptosis and angiogenesis: an evolving mechanism for fibrosis. FASEB J. 2013; 27: 3893-3901

19. Vénéreau E, Ceriotti C, Bianchi ME. DAMPs from cell death to new life. Front Immunol. 2015; 18:6-422

20. Hernández C, Huebener P, Schwabe RF. Damage-associated molecular patterns in cancer: A double-edged sword. Oncogene 2016; 35: 5931-5941

21. Benedict M, Zhang X. Non-alcoholic fatty liver disease: An expanded review. World J. Hepatol. 2017, 16, 715-732

22. Bataller R, Brenner DA. Liver Fibrosis. J Clin Invest. 2005; 115: 209-218

23. Stanger BZ. Cellular homeostasis and repair in the mammalian liver. Annu Rev Physiol 2015; 77:179-200

24. Fickert P, Wagner M. Biliary bile acids in hepatobiliary injury - What is the llink? J Hepatol. 2017; 67:619631

25. Fang YL, Chen H, Wang CL, et al. Pathogenesis of non-alcoholic fatty liver disease in children and adolescence: From "two hit theory" to "multiple hit model. World J Gastroenterol. 2018; 24: 2974-2983

26. Kochan K, Kus E, Szafraniec E, et al. Changes induced by non-alcoholic fatty liver disease in liver sinusoidal endothelial cells and hepatocytes: spectroscopic imaging of single live cells at the subcellular level. Analyst. 2017; 142:3948-3958

27. Takahashi Y, Fukusato T. Histopathology of nonalcoholic fatty liver disease/nonalcoholic steatohepatitis. World J Gastroenterol. 2014; 20: 15539-15548

28. Masarone M, Rosato V, Dallio M, et al. Role of Oxidative Stress in Pathophysiology of Nonalcoholic Fatty Liver Disease. Oxid Med Cell Longev. 2018; 2018: 9547613

29. Nassir F, Ibdah JA. Sirtuins and nonalcoholic fatty liver disease. World J Gastroenterol. 2016; 22: 1008410092

30. Handa P, Vemulakonda A, Kowdley KV, et al. Mitochondrial DNA from hepatocytes as a ligand for TLR9: Drivers of nonalcoholic steatohepatitis? World J Gastroenterol. 2016;22:6965-71.

31. Tu T, Calabro SR, Lee A, et al. Hepatocytes in liver injury: Victim, bystander, ¿or accomplice in progressive fibrosis? J Gastroenterol Hepatol. 2015; 30:1696-704. 
32. Garcia-Martinez I, Santoro N, Chen Y, et al. Hepatocyte mitochondrial DNA drives nonalcoholic steatohepatitis by activation of TLR9. J Clin Invest.2016;126:859-64.

33. Cai J, Zhang XJ, Li H. The Role of Innate Immune Cells in Nonalcoholic Steatohepatitis. Hepatology. 2019; 70:1026-1037.

34. Kolios G, Valatas V, Kouroumalis E. Role of Kupffer cells in the pathogenesis of liver disease. World J Gastroenterol. 2006; 12:7413-20

35. Dixon LJ, Barnes M, Tang H, et al. Kupffer Cells in the Liver. Compr Physiol. 2013; 3: 785-797

36. Huang W, Metlakunta A, Dedousis N, et al. Depletion of liver Kupffer cells prevents the development of diet -induced hepatic steatosis and insulin resistance. Diabetes 2010; 59:347 -357

37. Scott CL, Guilliams M. The role of Kupffer cells in hepatic iron and lipid metabolism. J Hepatol. 2018; 69:1197-119

38. Baffy G. Kupffer cells in non-alcoholic fatty liver disease: The emerging view. J Hepatol. 2009; 51: $212-223$.

39. Mendez-Sanchez N, Cruz-Ramon VC, Ramirez-Perez OL, et al. New Aspects of Lipotoxicity in Nonalcoholic Steatohepatitis. Int J Mol Sci. 2018 Jul;19. pii: E2034

40. Shoelson SE, Lee J, Goldfine AB. Inflammation and insulin resistance. J Clin Invest 2006; 116: 1793-801

41. Arguello G, Balboa E, Arrese M, et al. Recent insights on the role of cholesterol in non-alcoholic fatty liver disease. Biochim Biophys Acta. 2015; 1852:1765-78

42. Reid DT, Reyes JL, McDonald BA, et al. Kupffer Cells Undergo Fundamental Changes during the Development of Experimental NASH and Are Critical in Initiating Liver Damage and Inflammation. PLoS OnE. 2016; 11: e0159524.

43. Lanthier N, Molendi-Coste O, Cani PD, et al. Kupffer cell depletion prevents but has no therapeutic effect on metabolic and inflammatory changes induced by a high-fat diet. FASEB J 2011; 25: 4301-1.

44. Stienstra R, Saudale F, Duval C, et al. Kupffer cells promote hepatic steatosis via interleukin-1betadependent suppression of peroxisome proliferator-activated receptor alpha activity. Hepatology. 2010; 51:511-22

45. Kisseleva T. The Origin of Fibrogenic Myofibroblasts in Fibrotic Liver. Hepatology. 2017; 65: 1039-1043.

46. Xu J, Liu X, Koyama Y, et al. The types of hepatic myofibroblasts contributing to liver fibrosis of different etiologies. Front Pharmacol. 2014; 5: 167.

47. Iwaisako K, Jiang C, Zhang M, et al. Origin of myofibroblasts in the fibrotic liver in mice. Proc Natl Acad Sci U S A. 2014;111: E3297-E3305.

48. Marcher AB, Bendixen SM, Terkelsen MK, et al. Transcriptional regulation of Hepatic Stellate Cell activation in NASH. Sci Rep 2019;9: 2324.

49. Kocabayoglu P, Lade A, Lee YA, et al. $\beta$-PDGF receptor expressed by hepatic stellate cells regulates fibrosis in murine liver injury, but not carcinogenesis. J Hepatol. 2015; 63:141-7.

50. Coombes JD, Steve SC, Wing-Kin S, et al. Osteopontin is a proximal effector of leptin-mediated nonalcoholic steatohepatitis (NASH) fibrosis. Biochim Biophys Acta. 2016;1862: 135-144.

51. Saxena NK, Anania FA. Adipocytokines and hepatic fibrosis. Trends Endocrinol Metab. 2015; 26:153-61.

52. Liu C, Tao Q, Sun M, et al. Kupffer cells are associated with apoptosis, inflammation and fibrotic effects in hepatic fibrosis in rats. Lab Invest. 2010; 90:1805-1816.

53. Nieto N. Oxidative-stress and IL-6 mediate the fibrogenic effects of [corrected] Kupffer cells on stellate cells. Hepatology. 2006; 44:1487-501

54. Chu X, Jin Q, Chen H, et al. CCL20 is up-regulated in non-alcoholic fatty liver disease fibrosis and is produced by hepatic stellate cells in response to fatty acid loading. J Transl Med. 2018; 16: 108

55. Kim BM, Abdelfattah AM, Vasan R, et al. Hepatic stellate cells secrete Ccl5 to induce hepatocyte steatosis. Sci Rep. 2018; 8: 7499.

56. Liu Y, Liu H, Meyer C, et al. Transforming Growth Factor- $\beta$ (TGF- $\beta$ )-mediated Connective Tissue Growth Factor (CTGF) Expression in Hepatic Stellate Cells Requires Stat3 Signaling Activation. J Biol Chem. 2013; 288: 30708-30719

57. Furukawa F, Matsukai K, Tahashi Y, et al. p38 MAPK mediates fibrogenic signal through Smad3 phosphorylation in rat myofbroblasts. Hepatology. 2003; 38: 879-889

58. Kim KM, Han CY, Kim JY, et al. G 112 overexpression induced by miR-16 dysregulation contributes to liver fibrosis by promoting autophagy in hepatic stellate cells. J Hepatol. 2018; 68:493-504

59. Tacke F. Cenicriviroc for the treatment of non-alcoholic steatohepatitis and liver fibrosis. Expert Opin Investig Drugs. 2018;27:301-311 
60. Kmiéc Z. Cooperation of liver cells in health and disease. Adv Anat Embryol Cell Biol. 2001;161:111-X111.

61. Almeda-Valdes P, Uribe M, Méndez-Sánchez N, et al. The Role of Dendritic Cells in Fibrosis Progression in Nonalcoholic Fatty Liver Disease. Biomed Res Int. 2015;2015:768071.

62. Connolly MK, Bedrosian AS, Mallen-St Clair J, et al. In liver fibrosis, dendritic cells govern hepatic inflammation in mice via TNF-alpha. J Clin Invest. 2009; 119:3213-25.

63. Pradere, JP, Kluwe J, De Minicis S, et al. Hepatic macrophages but not dendritic cells contribute to liver fibrosis by promoting the survival of activated hepatic stellate cells in mice. Hepatology 2013; 58: 14611473.

64. Schuster S, Cabrera D, Arrese M, et al. Triggering and resolution of inflammation in NASH. Nat Rev Gastroenterol Hepatol. 2018; 15:349-364.

65. Van Herck MA, Weyler J, Kwanten WJ. The Differential Roles of T Cells in Non-alcoholic Fatty Liver Disease and Obesity. Front Immunol. 2019; 10: 82.

66. Bhattacharjee J, Kumar JM, Arindkar S, et al. Role of immunodeficient animal models in the development of fructose induced NAFLD. J Nutr Biochem. 2014; 25:219-26.

67. Narayanan S, Surette FA, Hahn YS. The Immune Landscape in Nonalcoholic Steatohepatitis. Immune Netw. 2016; 16: 147-158.

68. Ma X, Hua J, Mohamood AR, et al. A high-fat diet and regulatory $\mathrm{T}$ cells influence susceptibility to endotoxin-induced liver injury. Hepatology. 2007; 46:1519-29.

69. Tan Z, Qian X, Jiang R, et al. IL-17A plays a critical role in the pathogenesis of liver fibrosis through hepatic stellate cell activation. J Immunol. 2013; 191:1835-44.

70. Chackelevicius CM, Gambaro SE, Tiribelli C, et al. Th17 involvement in nonalcoholic fatty liver disease progression to non-alcoholic steatohepatitis. World J Gastroenterol. 2016; 22: 9096-9103.

71. Liu J, Yang P, Zuo G, et al. Long-chain fatty acid activates hepatocytes through CD36 mediated oxidative stress. Lipids Health Dis. 2018; 17: 153.

72. Ore A, Akinloye OA. Oxidative Stress and Antioxidant Biomarkers in Clinical and Experimental Models of Non-Alcoholic Fatty Liver Disease. Medicina. 2019; 55: 26.

73. Kolodziejczyk AA, Zheng D, Shibolet $\mathrm{O}$ et al. The role of the microbiome in NAFLD and NASH. EMBO Mol Med. 2019; 11: e9302.

74. Konturek PC, Harsch IA, Konturek K, et al. Gut-Liver Axis: How Do Gut Bacteria Influence the Liver? Med Sci . 2018; 6: 79.

75. Perdomo CM, Fruhbeck G, Escalada J. Impact of Nutritional Changes on Nonalcoholic Fatty Liver Disease Nutrients. 2019; 11: 677.

76. Farrell GC, Haczeyni F, Chitturi S. Pathogenesis of NASH: How Metabolic Complications of Overnutrition Favour Lipotoxicity and Pro-Inflammatory Fatty Liver Disease. Adv Exp Med Biol. 2018; 1061:19-44.

77. Neuschwander-Tetri BA. Hepatic lipotoxicity and the pathogenesis of nonalcoholic steatohepatitis: the central role of nontriglyceride fatty acid metabolites. Hepatology. 2010; 52:774-88.

78. Ibrahim H.S, Kohli R, Gores G.J. et al. Mechanisms of lipotoxicity in NAFLD and Clinical implications. J Pediatr Gastroenterol Nutr. 2011; 53:131-40.

79. Lebeaupin C, Vallée D, Hazari Y, et al. Endoplasmic reticulum stress signalli and the pathogenesis of nonalcoholic fatty liver disease. J Hepatol. 2018; 69:927-947.

80. Henkel A, Green R.M. The unfolded protein response in fatty liver disease. Semin Liver Dis. 2013; 33:321329.

81. Pessayre D. Role of mitochondria in non-alcoholic fatty liver disease. J Gastroenterol and Hepatol;2007:22:S20-S27.

82. Yang L, Roh Y.S, Song J, et al. Transforming growth factor beta signaling in hepatocytes participates in steatohepatitis through regulation of cell death and lipid metabolism in mice. Hepatology. 2013;59:483.495.

83. Hou B, Zhao Y, Qiang G, et al. Puerarin mitigates diabetic Hepatic Steatosis and Fibrosis by Inhibiting TGF$\beta$ Signaling Pathway Activation in Type 2 Diabetic Rats. Oxidative Medicine and cellular longevity. 2018; $1-13$.

84. Machado M.V, Diehl A.M. The hedgehog pathway in nonalcoholic fatty liver disease. Crit Rev Biochem Mol Biol. 2018;53:264-278.

85. Sircana A, Paschetta E, Musso G. Recent Insight into the Role of Fibrosis in Nonalcoholic SteatohepatitisRelated Hepatocellular Carcinoma. Int J Mol Sci. 2019; 20:1745. 
732

733

734

735

736

737

738

739

740

741

742

743

744

745

746

747

748

749

750

751

752

753

754

755

756

757

758

759

760

761

762

763

764

765

766

767

768

86. Zhou X, Wang P, Ma Z et al. Novel Interplay Between Sonic Hedgehog and Transforming Growth Factor$\beta 1$ in Human Nonalcoholic Steatohepatitis. Appl Immunohistochem Mol Morphol. 2019;67.

87. Kitade H, Chen G, Ni Y. Nonalcoholic Fatty Liver Disease and Insulin Resistance: New Insights and Potential New Treatments. 2017;9:387.

88. Angulo P, Alba LM, Petrovic LM, et al. Leptin, insulin resistance, and liver fibrosis inhuman nonalcoholic fatty liver disease. J Hepatol. 2004;41:943-9.

89. Dongiovanni P, Meroni M, Baselli GA, et al. Insulin resistance promotes Lysyl Oxidase Like 2 induction and fibrosis accumulation in non-alcoholic fatty liver disease. Clin Sci. 2017;131:1301-1315.

90. Chen Z, Yu R, Zhu S, et al. A vicious circle between insulin resistance and inflammation in nonalcoholic fatty liver disease. Lipids Health Dis. 2018;23:17:33

91. Higashi T, Friedman S.L, Hoshida Y. Hepatic stellate cells as key target in liver fibrosis. Adv Drug Deliv Rev. 2017:121:47-42

92. Wen H, Chao S, Cheng Ch. Homeostasis of Glucose and Lipid in Non-Alcoholic Fatty Liver Disease. Int J Mol Sci. 2019;20:298.

93. Konturek PC, Harsch IA, Konturek K, et al. Gut-Liver Axis: How Do Gut Bacteria Influence the Liver. Med Sci. 2018;6:79.

94. Meng X, Li S, Li Y, Gan RY, Li HB. Gut Microbiota's Relationship with Liver Disease and Role in Hepatoprotection by Dietary Natural Products and Probiotics. Nutrients. 2018;10:1457.

95. Chow MD, Lee YH, Guo GL. The role of bile acids in nonalcoholic fatty liver disease and nonalcoholic steatohepatitis. Mol Aspects Med. 2017;56:34-44.

96. Long SL, Gahan CGM, Joyce SA. Interaction between gut bacteria and bile in health and disease. Mol Aspects Med. 2017;56:54-65.

97. Sayin Sl, Wahlstrom A, Felin J, et al. Gut microbiota regulates bile acid metabolism by reducing the levels of tauro-beta-muricholic acid, a naturally occurring FXR antagonist. Cell Metab. 2013;17:225-35.

98. Han CY. Update on FXR Biology: Promising Therapeutic Target. Int J Mol Sci. 2018;19:2069.

99. Chen J, Thomsen M, Vitetta L. Interaction of gut microbiota with dysregulation of bile acids in the pathogenesis of nonalcoholic fatty liver disease and potential therapeutic implications of probiotics. J Cell Biochem. 2019;120:2713-2720.

100. Chávez-Talavera O, Tailleux A, Lefebvre P, et al. Bile Acid Control of Metabolism and Inflammation in Obesity, Type 2 Diabetes, Dyslipidemia, and Nonalcoholic Fatty Liver Disease. Gastroenterology 2017;152:1679-1694.

101. Arrese M, Cabrera D, Kalergis AM, Feldstein AE. Innate Immunity and Inflammation in NAFLD/NASH. Dig Dis Sci. 2016;61:1294-1303.

102. Campo L, Eiseler S, Apfel T, Pyrsopoulos N. Fatty Liver Disease and Gut Microbiota: A Comprehensive Update. J Clin Transl Hepatol. 2019;7:56-60.

103. Boursier J, Diehl AM. Nonalcoholic Fatty Liver Disease and the Gut Microbiome. Clin Liver Dis. 2016;20:263-75. 